\title{
Undetectable High-Sensitivity Troponin T as a Gatekeeper for Coronary Computed Tomography Angiography in Patients Suspected of Acute Coronary Syndrome
}

\author{
Murat Arslan ${ }^{a, b}$ Jeroen Schaap ${ }^{c}$ Pleunie P.M. Rood ${ }^{d}$ Koen Nieman ${ }^{a, b}$ \\ Ricardo P.J. Budde ${ }^{a, b}$ Bas M. van Dalen ${ }^{a, e}$ Mohamed Attrach ${ }^{b}$ Eric A. Dubois ${ }^{a, f}$ \\ Admir Dedic ${ }^{a, b}$

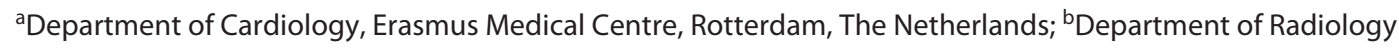 \\ and Nuclear Medicine, Erasmus Medical Centre, Rotterdam, The Netherlands; 'Department of Cardiology, \\ Amphia Ziekenhuis, Breda, The Netherlands; ${ }^{d}$ Department of Emergency Medicine, Erasmus Medical Centre,

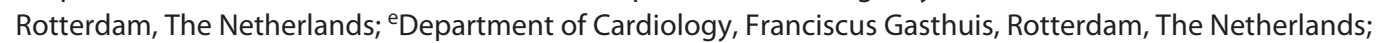 \\ fDepartment of Intensive Care, Erasmus University Medical Centre, Rotterdam, The Netherlands
}

\section{Keywords}

High-sensitivity troponin T · Coronary computed tomography angiography · Acute coronary syndrome ·

Emergency department

\begin{abstract}
Objectives: The aim of this study was to characterize the safety and efficiency of a strategy employing the limit of detection (LoD) of high-sensitivity troponin T (hs-TnT) as a gatekeeper for coronary computed tomography angiography (CCTA) in suspected acute coronary syndrome (ACS) patients in the emergency department (ED). Methods: We included suspected ACS patients who underwent CCTA and were evaluated with hs-TnT. Patients were categorized as below the LoD and at or above the LoD. The primary outcome was 30-day major adverse cardiac events (MACEs), defined as all-cause mortality, ACS, or coronary revascularization. Results: The study population consisted of 177 patients
\end{abstract}

karger@karger.com www.karger.com/crd

Karger $\stackrel{\text { ! }}{=}$

bOPEN ACCESS

\section{(C) 2021 The Author(s)}

Published by S. Karger AG, Basel

This is an Open Access article licensed under the Creative Commons Attribution-NonCommercial-4.0 International License (CC BY-NC) (http://www.karger.com/Services/OpenAccessLicense), applicable to the online version of the article only. Usage and distribution for commercial purposes requires written permission. (mean age $55 \pm 10$ years, 50.3\% women), and 16 (9.0\%) patients reached the primary outcome. None of the patients died, while 13 had an adjudicated diagnosis of ACS, and 3 underwent elective coronary revascularization. There were 77 patients (44\%) with an hs-TnT value below the LoD (MACEs; $n=1[1.3 \%])$ and 100 (56\%) with at or above the LoD levels (MACEs; $n=15$ [15\%]). None of 67 patients with an hs-TnT value below the LoD and $<50 \%$ stenosis on CCTA experienced MACEs. Out of the 10 patients with an hs-TnT value below the LoD and $\geq 50 \%$ stenosis on CCTA, 1 patient underwent elective percutaneous coronary revascularization. In patients with an hs-TnT value at or above the LoD, 74 patients had $<50 \%$ stenosis on CCTA, and 2 patients (3\%) were diagnosed with myocardial infarction without obstructive coronary artery disease confirmed on invasive angiography. Thirteen (50\%) patients with an hs-TnT value at or above the

The trial was registered at ClinicalTrials.gov (NCT01413282).
Correspondence to:

Murat Arslan, m.arslan@erasmusmc.nl 
LoD and $\geq 50 \%$ stenosis on CCTA experienced MACEs ( 11 ACS and 2 elective percutaneous coronary revascularizations). Conclusion: Our findings support that implementing the LoD of hs-TnT as a gatekeeper may reduce the need for CCTA in suspected ACS patients in the ED.

(c) 2021 The Author(s)

Published by S. Karger AG, Basel

\section{Introduction}

Patients with symptoms suggestive of an acute coronary syndrome (ACS) are evaluated on a day-to-day basis in emergency departments (ED) worldwide [1-3]. Physicians aim for an efficient diagnostic workup while maintaining patient safety $[2,4]$. High-sensitivity troponin T (hs-TnT) assay facilitates a more rapid and safe workup of suspected ACS patients [2,5]. For this reason, many sites worldwide have transitioned to these new biomarkers. Recent cohort studies have suggested that using the hs-TnT assay's limit of detection (LoD) as a cutoff permits the early and safe discharge of a considerable number of patients, thereby reducing downstream testing and resource utilization $[6,7]$.

Coronary computed tomography angiography (CCTA) is a valuable noninvasive imaging modality that has shown to accurately rule out ACS in the ED in large randomized trials [8-11]. However, CCTA necessitates radiation exposure to patients and requires certain logistic utilities in the ED. Implementing the LoD of hs-TnT as a gatekeeper for CCTA may provide a more efficient diagnostic workup, where CCTA can be reserved for a selected group of patients. The aim of this study was to characterize the safety and efficiency of a strategy employing the LoD of hsTnT followed by CCTA in the ED for the detection of 30day major adverse cardiac events (MACEs).

\section{Methods}

\section{Patient Population}

The multicenter randomized Better Evaluation of Acute Chest Pain with Computed Tomography Angiography trial compared a diagnostic strategy with early CCTA to standard optimal care in patients presenting to the ED with symptoms suggestive of ACS. The methods, including study designs, and inclusion and exclusion criteria have previously been published [8]. In this secondary analysis, we included patients who underwent CCTA of diagnostic image quality and for whom a baseline hs-TnT value was available. The study was conducted according to the principles of the Declaration of Helsinki, approved by the local institutional review boards (reference number 2011-071). All patients provided written informed consent. The trial was registered at ClinicalTrials.gov (NCT01413282).

\section{High-Sensitivity Troponin T}

Blood samples of each patient were obtained at the time of presentation to the ED. Cardiac troponin was measured with the fifth-generation Elecsys hs-TnT assay (Roche Diagnostics, Basel, Switzerland). The assay's limit of blank is $3 \mathrm{ng} / \mathrm{L}$, the LoD is 5 $\mathrm{ng} / \mathrm{L}$, and the 99 th percentile is $14 \mathrm{ng} / \mathrm{L}$ with a coefficient of variation $\leq 10 \%$ at $13 \mathrm{ng} / \mathrm{L}$. In the current sub-analysis, we only used baseline troponin values. Patients were categorized into 2 groups as follows: (1) below the $\operatorname{LoD}(<5 \mathrm{ng} / \mathrm{L})$ and (2) at or above the LoD ( $\geq 5 \mathrm{ng} / \mathrm{L})$.

\section{Coronary Computed Tomography Angiography}

Image acquisition was performed on 64-slice or newer computed tomography systems, using ECG-synchronized axial or spiral scan protocols combined with radiation minimizing measures, depending on local practices, available technology, and patient characteristics. Results of CCTA were locally evaluated and reported by certified radiologists with a minimum of 2 years of experience reading CCTA, thereby ensuring an expeditious workup. A combination of reconstruction methods was used, particularly the axial images and multiplanar reconstructions. The presence of coronary plaque and the degree of stenosis were visually assessed by eye-balling for each evaluable coronary segment. The degree of stenosis was divided on a patient level as nonobstructive coronary artery disease (CAD) ( $<50 \%$ stenosis) or obstructive CAD $(\geq 50 \%$ stenosis), in line with the cutoff for consideration of invasive angiography in recent guidelines for patients with acute chest pain [12].

\section{Clinical End Points}

The primary outcome was MACEs at 30 days after discharge from the hospital, which was defined as all-cause mortality, ACS, or coronary revascularization. ACS was defined as acute myocardial infarction (MI) or unstable angina according to the third universal definition of AMI [13]. MI with nonobstructive arteries (MINOCA) was defined as a MI, dependent on serial hs-TnT assessment with at least 1 value above the 99th percentile, with nonobstructive CAD on invasive coronary angiography (ICA) and no other clinically overt cause that could serve as an alternative diagnosis, based on ICA and echocardiography [14]. All clinical end points were adjudicated by 2 cardiologists who independently reviewed medical records of patients. Results of the CCTA were blinded to the cardiologists performing the event adjudication. Referral for ICA was at the discretion of the treating physician based on all available information, including the results of the CCTA.

\section{Statistical Analysis}

Continuous data are presented as mean \pm SD or median (interquartile ranges), and categorical data are presented as proportions (percentages). Differences between independent groups were compared using the independent samples $t$ test for continuous variables, and Fisher's exact test or the Pearson's $\chi^{2}$ test for categorical variables. All statistical analyses were performed using MedCalc Statistical Software version 18.10 (MedCalc Software bvba, Ostend, Belgium) and SPSS version 24.0 (IBM, Armonk, NY, USA). All tests were 2 -tailed, and a $p$ value $<0.05$ was considered statistically significant. 
Table 1. Baseline patient characteristics and outcomes at 30 days

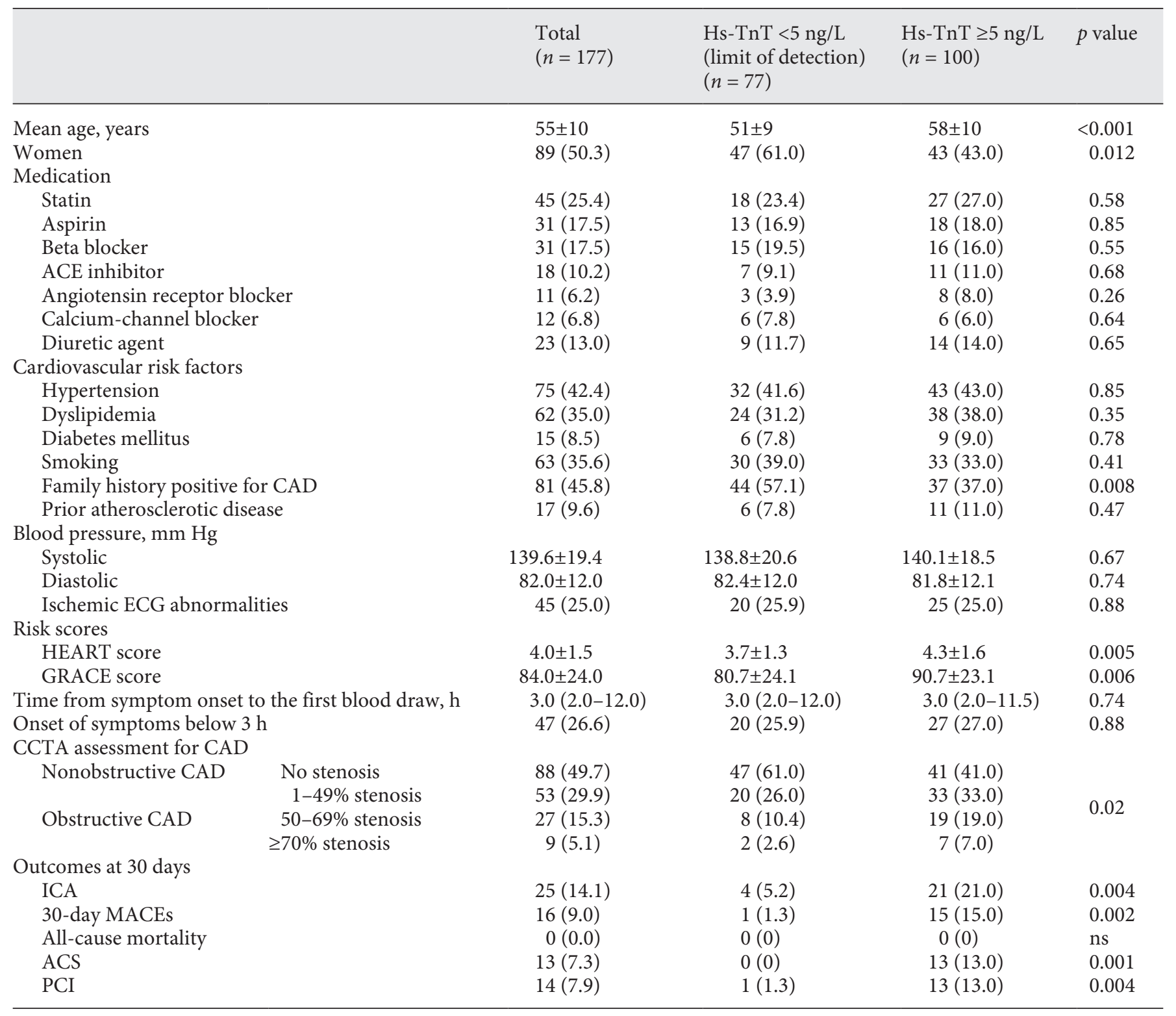

Values are mean $\pm \mathrm{SD}$, median (interquartile ranges), or $n(\%)$. ECG abnormalities are defined as Q-wave or ST-T-segment alterations suggestive of ischemia; ACS, acute coronary syndrome; ACE, angiotensin-converting enzyme; CAD, coronary artery disease; CCTA, coronary computed tomography angiography; ECG, electrocardiogram; Hs-TnT, high-sensitivity troponin T; PCI, percutaneous coronary intervention; ns, nonsignificant; ICA, invasive coronary angiography; HEART, history, ECG, age, risk factors, and initial troponin; GRACE, The Global Registry of Acute Coronary Events; MACEs, major adverse cardiac events.

\section{Results}

A total of 177 patients had a baseline hs-TnT measurement and a CCTA of diagnostic image quality and were eligible for inclusion. Baseline characteristics of included patients are shown in Table 1 . The mean age was $55 \pm 10$ years, and the proportion of women was $50.3 \%$. Patients with hs-TnT at or above LoD were more often male and older. In patients with hs-TnT levels at or above the LoD, the median hs-TnT value was 7 [5-10] ng/L. The median time from symptom onset to the first blood draw was 3.0 (2.0-12.0) h. None of the patients died within 30 days. 
Fig. 1. Frequency of 30-day MACEs according to baseline hs-TnT values and stenosis severity on CCTA. Nonobstructive $\mathrm{CAD}$ was defined as $<50 \%$ stenosis on CCTA, whereas obstructive CAD was defined as $\geq 50 \%$ stenosis on CCTA. MACEs is defined as all-cause mortality, ACS, or coronary revascularization. CAD, coronary artery disease; CCTA, coronary computed tomography angiography; Hs-TnT, high-sensitivity cardiac troponin T; MACEs, major adverse cardiac events; ACS, acute coronary syndrome.

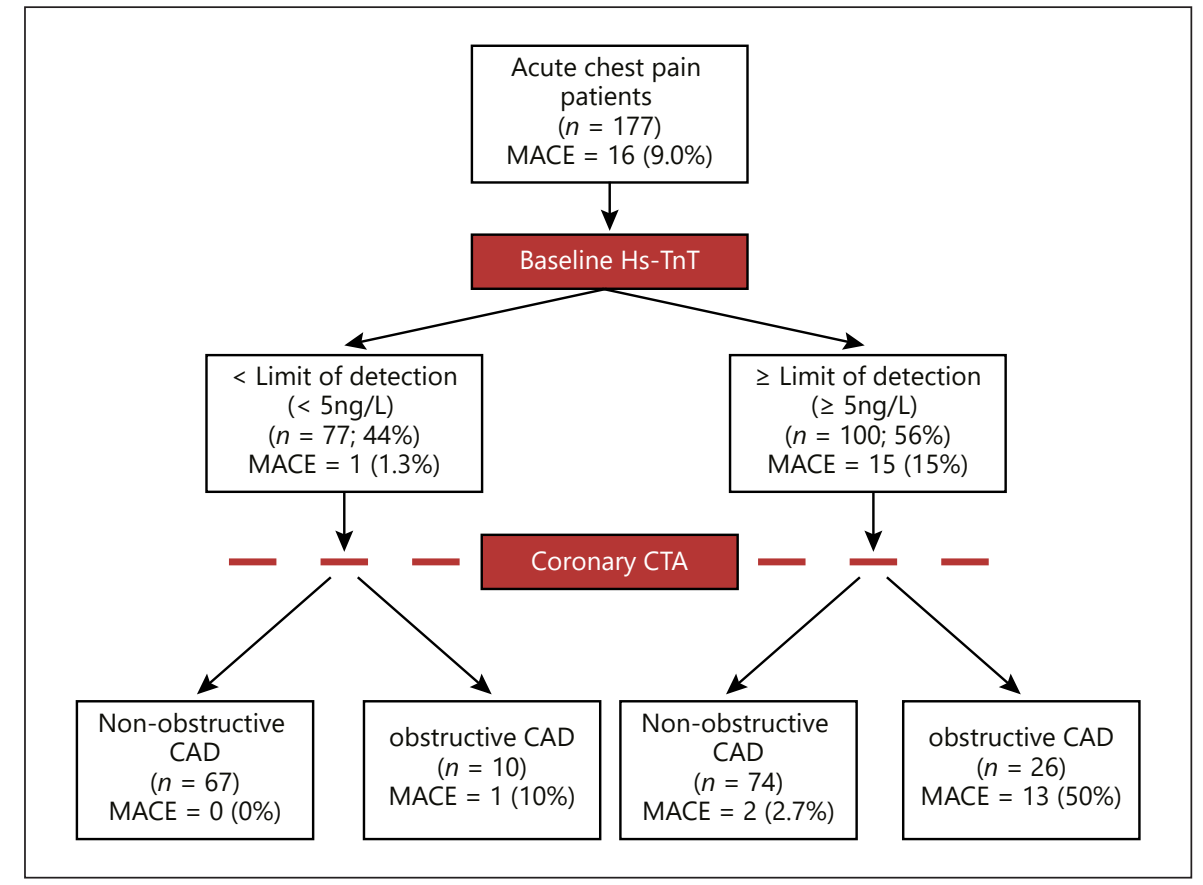

Thirteen patients had an adjudicated diagnosis of ACS, and 3 patients underwent coronary revascularization for stable CAD within 30 days (Table 1 ).

\section{$H s-T n T$ and CCTA}

The results of CCTA showed that $141(79.7 \%)$ patients had nonobstructive CAD, and 36 (20.3\%) patients had obstructive CAD (Table 1). The MACEs rate in patients with hs-TnT below the LoD $(n=77 ; 44 \%)$ and at or above the $\operatorname{LoD}(n=100 ; 56 \%)$ was $1.3 \%(n=1)$ and $15 \%(n=$ 15), respectively (Fig. 1). Below the LoD, 67 (87.0\%) patients had nonobstructive CAD on CCTA, and 10 (13.0\%) patients had obstructive CAD on CCTA. Out of the 67 patients, with an hs-TnT value below the LoD and nonobstructive CAD on CCTA, 0 patients were referred to ICA, and 0 patients had 30-day MACEs. Out of the 10 patients, with an hs-TnT value below the LoD and obstructive CAD on CCTA, 4 patients underwent ICA, of whom 1 underwent elective percutaneous coronary revascularization. The remaining 6 patients were not referred for ICA, as they were deemed to have lesions for which PCI was not indicated (e.g., distal branches or lesions in small side branches). These patients were discharged from the ED and received further evaluation and medical treatment at the outpatient clinic.

At or above the LoD, 74 (74.0\%) patients had nonobstructive CAD on CCTA, and $26(26.0 \%)$ patients had obstructive CAD on CCTA. Out of the 74 patients, with a hs-TnT value at or above the $\mathrm{LoD}$ and nonobstructive CAD on CCTA, 3 patients underwent ICA, of whom 2 patients (3\%) experienced MACEs within 30 days. Both patients had nonobstructive coronary artery disease on invasive angiography and were diagnosed as MINOCA. Out of the 26 patients, with an hs-TnT value at or above the LoD and obstructive CAD on CCTA, 18 (69\%) patients were referred to ICA. Thirteen (50\%) patients had a MACE within 30 days, of whom 11 patients were diagnosed with ACS, and 2 patients underwent elective percutaneous coronary revascularization.

\section{Discussion}

In the current study, we explored the safety and efficiency of a strategy employing the LoD of hs-TnT as a gatekeeper for CCTA in suspected ACS patients in the ED and report several important findings. First, no patient with a baseline hs-TnT value below the LoD had ACS within 30 days. Second, in the ED setting, it may be sensible to omit performing CCTA regularly in suspected ACS patients with a baseline troponin value below the LoD, considering the low incidence of events. Third, patients with a baseline value of hs-TnT above LOD had a $>10$-fold higher incidence of 30-day MACEs. In this 
group, CCTA could accurately discriminate between patients with and without a coronary related condition.

\section{Hs-TnT below the LoD}

Several prospective studies have shown a promising role of a single baseline hs-TnT value to discriminate between patients with and without AMI $[6,7,15]$. Recently, investigators from the HISTORIC trial showed that an hs-TnT value below the LoD safely rules out the occurrence of cardiac death and AMI within 30 days, if the patient has had symptoms for at least $2 \mathrm{~h} \mathrm{[16].} \mathrm{The} \mathrm{propor-}$ tion of patients with baseline hs-TnT below the LoD is not trivial, in our study approaching half of the population (44\%), which is in line with previous studies [6, 7]. Only $1(1.3 \%)$ patient with an hs-TnT value below the LoD experienced an adverse event, namely an elective percutaneous coronary intervention. This was correctly identified by CCTA; however, because of the low incidence and questionable prognostic implications, it may be sensible to omit performing CCTA in the ED setting regularly in suspected ACS patients with a baseline troponin value below the LoD. Further testing in an outpatient setting remains an attractive option.

Several factors should be considered when using a single baseline hs-TnT to discharge patients from the ED. First, considering the time dependency of troponin release, physicians should be cautious with the use of single baseline hs-TnT values for clinical decision-making in early presenters ( $<3 \mathrm{~h}$ of chest pain onset) as late increases in cardiac troponin can occur in these patients. Currently, in early presenters it is recommended to perform a second blood draw $3 \mathrm{~h}$ after symptom onset [16]. Second, the decision for early discharge should not solely be based on troponin levels but on the whole clinical picture as troponins are only a part of the diagnostic workup for suspected ACS patients. Third, the use of validated rapid risk stratification tools, such as the history, ECG, age, risk factors, and initial troponin score [17], 2-h accelerated diagnostic protocol to assess patients with chest pain symptoms using contemporary troponins as the only biomarker [18], and Emergency Department Assessment of Chest Pain Score [19] pathways can aid the physician in the assessment of suspected ACS patients. Patients can be discharged safely if there are no alarming findings and other life-threatening conditions are excluded. The USA and Europe differ in the way that results for the Hs-TnT assay are reported in clinical practice. Per USA, Food and Drug Administration regulations results less than the limit of quantification (LoQ), which is $6 \mathrm{ng} / \mathrm{L}$, are not reported in the USA. In our study population, 103 patients ( $58 \%$ of the

Undetectable Hs-TnT as a Gatekeeper for

CCTA in Patients Suspected of ACS total population) had hs-TnT levels below the LoQ, with a MACEs rate of $2.9 \%(n=3)$, of whom all 3 underwent elective PCI. Thus, the MACEs rate would increase when using the LoQ instead of the LoD. Nevertheless, we believe that the safety of such a strategy would not be compromised considering that none of these patients died or had an ACS within 30 days of discharge from the hospital.

\section{Patients with Baseline Hs-TnT at or above the LoD}

The first hs-TnT at the time of presentation enables early risk stratification, with a MACEs rate of $15.0 \%$ in patients with baseline hs-TnT at or above the LoD in our study. Implementing CCTA in patients with baseline hs$\mathrm{TnT}$ at or above the LoD enables further risk stratification as $50 \%$ of patients with obstructive CAD on CCTA experienced MACEs compared to 3\% with nonobstructive CAD on CCTA (both classified as MINOCA) [13]. Interestingly, no patient underwent coronary artery bypass grafting, which was most likely influenced by local revascularization practice and partially due to sheer coincidence. The introduction of high-sensitivity troponins has changed our perspective on MI as these new biomarkers quickly and more accurately detect acute myocardial injury of various origins $[20,21]$. More and more patients with elevated hs-TnT have nonobstructive CAD on invasive angiography $[22,23]$. Some of these are categorized as MINOCA, while others have a nonischemic reason altogether for their elevated cardiac markers. Importantly, noninvasive imaging with CCTA is a trustworthy gatekeeper, also in our study, to safely defer patients from invasive angiography who do not need it.

\section{Limitations}

Several limitations should be mentioned. The current study is a secondary analysis of patients suspected of ACS that underwent CCTA in the ED, for which prior specific power analyses were not performed and should therefore be regarded as hypothesis generating. In the main Better Evaluation of Acute Chest Pain with Computed Tomography Angiography trial, in and exclusion criteria were set to ensure a low-risk suspected ACS population without any contraindications that prohibited them from undergoing CCTA, which may affect the generalizability of these results to a real-world acute chest pain population. Furthermore, patients were enrolled in both community hospitals and tertiary medical centers, where patients may present with varying a priori risk of ACS, leading to a more heterogeneous study population. Quality control protocols for the hs-TnT assays used at the various sites during the study period were not at our disposal and may 
have varied between these sites, thereby influencing our results. The study population consisted of patients in whom results of CCTA were used as part of their clinical workup which in turn might have introduced a workup bias. A substantial number of patients did not have a second troponin blood draw, which prohibited us from performing further meaningful analysis into the kinetics of troponin release in the entire population. In our study, a quarter of the patients presented to the ED within $2 \mathrm{~h}$ of symptom onset. A second sample should be retrieved in these patients $3 \mathrm{~h}$ after symptom onset, considering the time dependency of troponin release. While discharge seems feasible in patients with representative hs-TnT below the LoD, further screening in an outpatient setting should be considered, where CCTA remains an attractive diagnostic option for selected patients.

\section{Conclusion}

Our results support that implementing the LoD of hsTnT as a gatekeeper in suspected ACS patients may reduce the need for CCTA in the ED. In our cohort of patients with a baseline hs-TnT value at or above the LoD, our findings indicate that CCTA may discriminate patients with and without a coronary related condition.

\section{Statement of Ethics}

The study was conducted according to the principles of the Declaration of Helsinki and approved by the local institutional review boards (reference number 2011-071). All patients provided written informed consent. The trial was registered at ClinicalTrials.gov (NCT01413282).

\section{Conflict of Interest Statement}

Koen Nieman was supported by a grant from the Dutch Heart Foundation (NHS 2014T061) and received grants from Siemens Medical Solutions, GE Healthcare, Bayer Healthcare, and HeartFlow outside the submitted work. All the other authors report no relationships relevant to the contents of this study to disclose.

\section{Funding Sources}

This work was supported by a grant from Erasmus MC and a research grant from the Erasmus MC Thorax Foundation (project grant B4). The sponsor had no role in study design; in the collection, analysis, and interpretation of data; in writing the report; and in the decision to submit the article for publication.

\section{Author Contributions}

Murat Arslan: conceptualization, formal analysis, project administration, visualization, and writing - original draft. Jeroen Schaap and Pleunie P.M. Rood: data curation, investigation, project administration, resources, and writing - review and editing. Koen Nieman: Conceptualization, data curation, formal analysis, funding acquisition, investigation, methodology, project administration, resources, and writing - review and editing. Ricardo P.J. Budde, Bas M. van Dalen, and Mohamed Attrach: data curation, investigation, resources, and writing - review and editing. Eric A. Dubois: conceptualization, data curation, formal analysis, funding acquisition, resources, supervision, visualization, writing - original draft, and writing - review and editing. Admir Dedic: conceptualization, data curation, formal analysis, funding acquisition, investigation, methodology, project administration, resources, supervision, visualization, writing - originaldraft, and writing - review and editing.

\section{References}

1 Goodacre S, Cross E, Arnold J, Angelini K, Capewell S, Nicholl J. The health care burden of acute chest pain. Heart. 2005;91(2):229-30.

2 Reichlin T, Hochholzer W, Bassetti S, Steuer S, Stelzig C, Hartwiger S, et al. Early diagnosis of myocardial infarction with sensitive cardiac troponin assays. N Engl J Med. 2009; 361(9):858-67.

3 Than M, Cullen L, Reid CM, Lim SH, Aldous S, Ardagh MW, et al. A 2-h diagnostic protocol to assess patients with chest pain symptoms in the Asia-Pacific region (ASPECT): a prospective observational validation study. Lancet. 2011;377(9771):1077-84.

4 Mokhtari A, Borna C, Gilje P, Tydén P, Lindahl B, Nilsson HJ, et al. A 1-h combination algorithm allows fast rule-out and rule-in of major adverse cardiac events. J Am Coll Cardiol. 2016;67(13):1531-40.

5 Giannitsis E, Kurz K, Hallermayer K, Jarausch J, Jaffe AS, Katus HA. Analytical validation of a high-sensitivity cardiac troponin $\mathrm{T}$ assay. Clin Chem. 2010;56(2):254-61.

6 Bandstein N, Ljung R, Johansson M, Holzmann MJ. Undetectable high-sensitivity cardiac troponin $\mathrm{T}$ level in the emergency department and risk of myocardial infarction. J Am Coll Cardiol. 2014;63(23):2569-78.

7 Rubini Giménez M, Hoeller R, Reichlin T, Zellweger C, Twerenbold R, Reiter M, et al. Rapid rule out of acute myocardial infarction using undetectable levels of high-sensitivity cardiac troponin. Int J Cardiol. 2013;168(4) 3896-901.
8 Dedic A, Lubbers MM, Schaap J, Lammers J, Lamfers EJ, Rensing BJ, et al. Coronary CT angiography for suspected ACS in the era of high-sensitivity troponins: randomized multicenter study. J Am Coll Cardiol. 2016;67(1): $16-26$.

9 Finck T, Hardenberg J, Will A, Hendrich E, Haller B, Martinoff S, et al. Ten-year followup after coronary computed tomography angiography in patients with suspected coronary artery disease. JACC Cardiovasc Imaging. 2019:12(7 Pt 2):1330-38.

10 Hoffmann U, Truong QA, Schoenfeld DA, Chou ET, Woodard PK, Nagurney JT, et al. Coronary CT angiography versus standard evaluation in acute chest pain. $\mathrm{N}$ Engl J Med. 2012;367(4):299-308. 
11 Schlett CL, Banerji D, Siegel E, Bamberg F, Lehman SJ, Ferencik M, et al. Prognostic value of CT angiography for major adverse cardiac events in patients with acute chest pain from the emergency department: 2-year outcomes of the ROMICAT trial. JACC Cardiovasc Imaging. 2011;4(5):481-91.

12 Cury RC, Abbara S, Achenbach S, Agatston A, Berman DS, Budoff MJ, et al. CADRADS(TM) coronary artery disease: reporting and data system. An expert consensus document of the Society of Cardiovascular Computed Tomography (SCCT), the American College of Radiology (ACR) and the North American Society for cardiovascular imaging (NASCI). Endorsed by the American College of Cardiology. J Cardiovasc Comput Tomogr. 2016 Jul-Aug;10(4):269-81.

13 Thygesen K, Alpert JS, Jaffe AS, Chaitman BR, Bax JJ, Morrow DA, et al. Fourth universal definition of myocardial infarction (2018). J Am Coll Cardiol. 2018;72(18):2231-64.

14 Tamis-Holland Jacqueline E, Jneid H, Reynolds Harmony R, Agewall S, Brilakis Emmanouil S, Brown Todd M, et al. Contemporary diagnosis and management of patients with myocardial infarction in the absence of obstructive coronary artery disease: a scientific statement from the American Heart Association. Circulation. 2019;139(18):e891-908.

15 Body R, Carley S, McDowell G, Jaffe AS, France M, Cruickshank K, et al. Rapid exclusion of acute myocardial infarction in patients with undetectable troponin using a high-sensitivity assay. J Am Coll Cardiol. 2011;58(13): 1332-9.

16 Collet JP, Thiele H, Barbato E, Barthélémy O, Bauersachs J, Bhatt DL, et al. 2020 ESC Guidelines for the management of acute coronary syndromes in patients presenting without persistent ST-segment elevation. Eur Heart J. 2021;42(14):1289-367.

17 Backus BE, Six AJ, Kelder JC, Mast TP, van den Akker F, Mast EG, et al. Chest pain in the emergency room: a multicenter validation of the HEART Score. Crit Pathw Cardiol. 2010; 9(3):164-9.

18 Than M, Aldous S, Lord SJ, Goodacre S, Frampton CM, Troughton R, et al. A 2-hour diagnostic protocol for possible cardiac chest pain in the emergency department: a randomized clinical trial. JAMA Intern Med. 2014;174(1):51-8.

19 Than MP, Pickering JW, Aldous SJ, Cullen L, Frampton CM, Peacock WF, et al. Effectiveness of EDACS versus ADAPT accelerated di- agnostic pathways for chest pain: a pragmatic randomized controlled trial embedded within practice. Ann Emerg Med. 2016;68(1):93102.e1.

20 Shah ASV, Anand A, Strachan FE, Ferry AV, Lee KK, Chapman AR, et al. High-sensitivity troponin in the evaluation of patients with suspected acute coronary syndrome: a stepped-wedge, cluster-randomised controlled trial. Lancet. 2018;392(10151):919-28.

21 Thygesen K, Alpert JS, Jaffe AS, Chaitman BR, Bax JJ, Morrow DA, et al. Fourth universal definition of myocardial infarction (2018). Eur Heart J. 2019;40:237-69.

22 Pasupathy S, Air T, Dreyer RP, Tavella R, Beltrame JF. Systematic review of patients presenting with suspected myocardial infarction and nonobstructive coronary arteries. Circulation. 2015;131(10):861-70.

23 Smilowitz NR, Mahajan AM, Roe MT, Hellkamp AS, Chiswell K, Gulati M, et al. Mortality of myocardial infarction by sex, age, and obstructive coronary artery disease status in the ACTION registry-GWTG (acute coronary treatment and intervention outcomes network registry-get with the guidelines). Circ Cardiovasc Qual Outcomes. 2017; 10(12):e003443. 